under the negative emotional challenge, cognitive DLPFC activation response was reduced with lamotrigine and risperidone. This explains the possibility that cognitive enhancers may be needed post-mood stabilization, based on medications used or to improve the residual attention difficulties intrinsic to PBD (Pavuluri et al, 2009). Furthermore, prognostic markers of clinical outcome began to emerge, with greater amygdala activity at baseline being a marker for poor outcome in the case of risperidone (possibly involving greater effort to subdue the amygdala), whereas greater MPFC activity was a marker for a good outcome (a sign of greater deployment of higher cortical region) in the case of DVPX, on direct comparison of these medication groups. Overall, these results indicated three things: (1) negative or angry stimuli were more successful in eliciting drug-related activity than happy or neutral faces in probing group differences; (2) medications differentially engaged the brain circuitry based on neurochemistry and tasks; and (3) state $v s$ trait: with all medications, the amygdala showed reduced activity from baseline along with recovery from manic state, but remained active relative to that of HC (trait marker), while the PFC regions were normalized with mood stabilization (state marker). However, long-term treatment for at least 4 months with a standardized algorithm led to increased amygdala connectivity (Wegbreit et al, 2011) in the affective circuitry and normalized subcortical activity (Yang et al, 2013).

In summary, in PBD, multiple domains are malfunctioning at baseline. Combination of treatments such as coupling mood stabilizers with antipsychotics for severe episodes of mania may be useful as each type of medication engages different brain circuits.

\footnotetext{
Mani Pavuluri ${ }^{1}$

${ }^{1}$ Pediatric Mood Disorders Program, Department of Psychiatry, University of Illinois at Chicago, Chicago, IL, USA

E-mail: Mpavuluri@psych.uic.edu
}

FUNDING AND DISCLOSURE

Dr Pavuluri receives or has received grant support from the following sources: National Institutes of Health (NIH), the National Institute of Mental Health, the National Alliance for Research on Schizophrenia and Depression (NARSAD), the American Foundation for Suicide Prevention, and the Marshall Reynolds Foundation. She is the recipient of the BergerColbeth Term Chair in Child Psychiatry and participated in the Otsuka Pharmaceuticals National Advisory Board meeting once.

Chang KD, Wagner C, Garrett A, Howe M, Reiss A (2008). A preliminary functional magnetic resonance imaging study of prefrontal-amygdalar activation changes in adolescents with bipolar depression treated with lamotrigine. Bipolar Disorder 10: 426-431.

Mayanil T, Wegbreit E, Fitzgerald J, Pavuluri MN (2011). Emerging biosignature of brain function and intervention in pediatric bipolar disorder. Minerva Pediatr 63: 183-200.

Pavuluri MN, West A, Hill K, Jindal K, Sweeney JA (2009). Neurocognitive Function in pediatric bipolar disorder: three-year follow-up shows cognitive development lagging behind healthy youth. J Am Acad Child Adolesc Psychiatry 48: 299-307.

Pavuluri MN. Neurobiology of bipolar disorder in youth in bipolar disorder in youth. In: Strakowski S, Adler C, Del Bello M (eds). Chapter 13, 1st edn. Oxford Press: NYC.

Wegbreit E, Ellis J, Nandam A, Fitzgerald J, Passarotti A, Pavuluri MN et al. (2011). Amygdala Functional Connectivity Predicts Pharmacotherapy Outcome in Pediatric Bipolar Disorder. Brain Connect 1: 411-422. Yang H, Lu L, Wu M, Stevens M, Wegbreit E, Fitzgerald J et al. (2013). Time Course of Recovery Showing Initial Prefrontal Cortex Changes at 16 weeks times. J Affect Disorders 13: 123-127.

Neuropsychopharmacology Reviews (2015) 40, 249-25I; doi: 1 0.1038/npp.2014.229

\section{Mutual Information in a MEG Complexity Measure Suggests Regional Hyper-Connectivity in Schizophrenic Probands}

Abnormalities of regional brain functional connectivity have been suggested for the syndromes of schizophrenia since the origin of the name using the Greek roots: skhizein (to split) and phren (mind) by Eugen Bleuler in 1908 (Kuhn, 2004). The neuropsychologist Norman Geschwind generalized this pathophysiological concept in his classic papers about what he called the disconnexion syndromes (Geschwind, 1965). He suggested that deficits in higher functions resulted from the disruption of pathways involving the signal relay functions of the association cortices. Discontinuities in white matter observed in diffusion tensor imaging and inferences from fMRI have been interpreted as evidence for cortical network disconnections in patients with schizophrenia (Bullmore et al, 1997).

More dynamical approaches to functional pathophysiological connections between brain regions have assessed their nearly simultaneous mutual similarities in frequency, wavelength, and phase (Uhlhaas and Singer, 2006). In addition, analysis of the envelope of oscillatory activity has yielded additional measures of connectivity. However, the brain does not operate by copying information from one region to another. The more general approach is to observe how broadband electrophysiological activity in one region shares information with other regions. To accomplish this we have expanded on our previous work using nonlinear dynamical measures of complexity for cortical activity (Robinson et al, 2012; Mandell, 2013).

A unique non-parametric measure of dynamical complexity, the symbolic rank vector approach (Robinson et al, 2012), was invoked to characterize the activity of the beamformer localized, time-dependent sources of the resting 275-channel whole-head magnetoencephalogram (MEG). MEG is the magnetic counterpart of EEG and has the advantage of yielding measures of cortical activity that are nearly independent of intervening conductivities. The source time series for an array of voxel locations is estimated from the multi-channel measurements using a linearly constrained minimum variance beamformer (Robinson and Rose, 1993). Each voxel time series is then converted to a symbolic time series by ranking their source magnitudes within a short $n$-place moving window. A measure of shared information, symbolic mutual information (SMI), is then computed for all voxel pairs from the probability of occurrence of their symbolic states (see Kraskov et al, 2004).

Subjects were recruited nationwide as part of an ongoing family study of schizophrenia at the Clinical Brain 

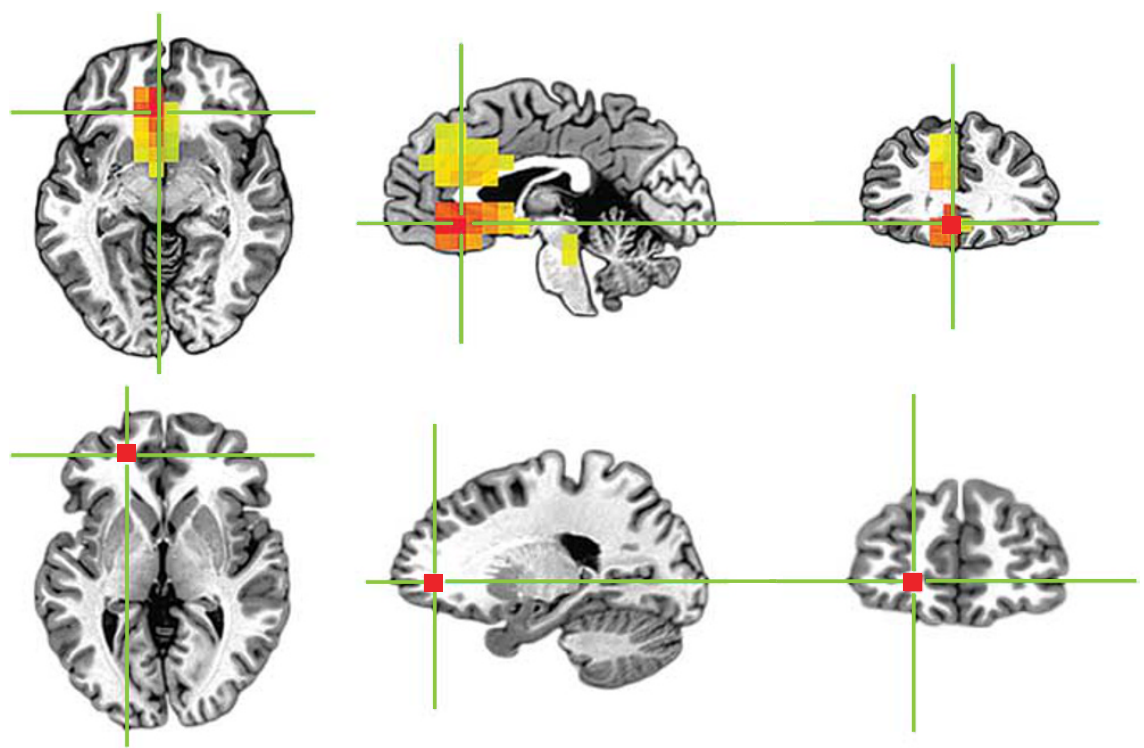

Figure 1. Group difference (top) between schizophrenics $(n=15)$ and normal control subjects $(n=14)$ for symbolic mutual information during rest with FDR threshold $q<0.01$. The seed location is shown at the bottom. This is one example of hyperconnectivity in rostral prefrontal cortex.

Disorders Branch Sibling Study (Protocol 95-M-0150) at the NIH and supported by the NIMH Intramural Research Program. MEG was collected under Protocol 99-M-0172. SMI analysis was applied to resting MEG data from 15 proband schizophrenics (10 M/5 F, 20-45 years) and 14 normal control subjects ( $5 \mathrm{M} / 9 \mathrm{~F}, 19-36$ years). The schizophrenic subjects all met the DSM-IV-TR criteria and were medicated to $\sim 500 \mathrm{CPZ}$ equivalents. Figure 1 illustrates a typical example of the network differences, showing characteristically higher values for SMI in schizophrenic than in normal controls, suggesting hyper-connectivity in rostral prefrontal cortex for short-range connections. Hypo-connectivity was also observed for long-range connections to lateral prefrontal cortical regions. The left prefrontal lobe location of this hyperconnectivity example is consistent with the literature implicating this area in the primary thought disorder, ambivalence, and thought-blocking characteristics of this patient group (Kuhn, 2004).

These preliminary findings are subject to refinement by incorporation of cofactors such as diagnostic scale, medication, and gender.The SMI measure of shared information is not dependent upon task-related activity and may be applicable to assessing psychiatric disorders and their response to psychopharmaceuticals.

\section{ACKNOWLEDGEMENTS}

This study was supported by the National Institute of Mental Health, NIMH Intramural Research Program (Robinson), and the Fetzer-Franklin Trust of the Fetzer Foundation (Mandell).

\section{Stephen E Robinson ${ }^{1}$ and}

Arnold J Mandell ${ }^{2}$

${ }^{1}$ MEG Core Facility, NIMH, Bethesda, MD, USA;

${ }^{2}$ Department of Psychiatry, UCSD School of

Medicine, San Diego, CA, USA

E-mail: robinsonse@mail.nih.gov

\section{FUNDING AND DISCLOSURE}

The authors declare no conflict of interest.

Bullmore E, Frangou S, Murray R (1997). The dysplastic net hypothesis: integration of developmental and dysconnectivity theories of schizophrenia. Schiz Res 28: 143-156.

Geschwind N (1965). Disconnexion syndromes in animals and man. I. Brain 88: 237-294.

Kraskov A, Stögbauer H, Grassberger P (2004). Estimating mutual information. Phys Rev E 69: 066138.

Kuhn R (2004). Eugen Bleuler's concepts of psychopathology. History Psychiatry 15: 361-366.

Mandell AJ (2013). Can a metaphor of physics contribute to neuroscience research; intermittent turbulent eddies in brain magnetic fields. Chaos Solitons Fractals 55: 95-101.
Robinson SE, Mandell AJ, R. Coppola R (2012). Spatiotemporal imaging of complexity. Front Comput Neurosci 6: 101-114.

Robinson SE, Rose DF (1993). Current source image estimation by spatially filtered MEG. In: Hoke M, Erné SN, Okada YC, Romani GL (eds). Biomagnetism: Clinical Aspects. Elsevier Science Publishers B. V.: Amsterdam, pp 761-765.

Uhlhaas P, Singer W (2006). Neural synchrony in brain disorders: relevance for cognitive dysfunctions and pathophysiology. Neuron 52 : 155-168.

Neuropsychopharmacology Reviews (2015) 40, 25I-252; doi: 10.1038/npp.2014.217

\section{Copy Number Variation in Schizophrenia}

Copy number variation contributes substantially to human evolution, normal phenotypic variation, and human disease (Malhotra and Sebat, 2012). To date, thousands of different genomic duplications and deletions, each spanning hundreds to millions of basepairs, have been mapped genomewide, and collectively account for a significant fraction of human genetic variation. By reorganizing broad swaths of DNA, structural mutations potentially create new genes and regulatory motifs while also disrupting established genes.

Copy number variants (CNVs) tend to occur in regions rich in genes, segmental duplications, and mobile elements(Malhotra and Sebat, 2012). Some arise repeatedly as de novo mutations in genomic 'hotspots.' The genomic architecture of hotspots is typically characterized by large repeated segments that increase the risk for errors in replication, a process known as nonallelic homologous recombination. If a $\mathrm{CNV}$ is pathogenic, it may persist for only a few generations given negative selection. At the same time, in each generation many de novo CNVs are introduced given the highly repetitive nature of the human genome.

The importance of rare copy number mutations for neuropsychiatric disease is now well-established (Malhotra and Sebat, 2012). Our group was the first to demonstrate that individuals with 